\title{
Editorial: Older Driver Research. Evidence to Guide Change*
}

\section{Background}

In the 20th and 21st centuries, the ability to drive an automobile has become critically interlinked with community mobility. Community infrastructure, housing patterns, and even our walkways are centred around roadways designed for automobiles. Driving is an expectation for a person of any age, and the loss of the ability to drive reflects loss of autonomy, health, and community access. In his study of Canadian seniors' transportation habits, Turcotte (2012) found that seniors are highly dependent on their motor vehicles, the number of senior drivers over age 65 will continue to increase, and "the majority of seniors have no intention of moving and plan to remain where they live as long as possible" (p. 15). As the population ages, the proportion of older drivers on the road will increase. This may have implications at many levels including policy, health, and public safety, since past and current approaches regarding driving have focused on younger drivers.

Health and aging have not historically been areas of focus of policy and infrastructure development for driving. Traditionally, emphasis has been placed on driving skill and knowledge, with the presumption that health and physical and cognitive functioning are intact. However, it is well-known that diseases and chronic medical conditions can affect the ability to drive (Charlton et al., 2010; Marshall, 2008), and this impact will likely be greater with the increase in older drivers. Historically, it has been presumed that an evaluation of health status can fairly assess one's fitness to continue driving. This simplistic approach, however, is not realistic since individuals can have multiple diagnoses, differing severities of disease, and functional impairments that may affect their driving ability.

Driving is a functional activity that fits well in the International Classification of Functioning, Disability and Health (ICF) developed by the World Health Organization (2013). Driving is impacted not only by health factors (e.g., physical, cognitive, and behavioural impairments) but also by personal factors (e.g., driving experience, need to drive), environmental factors (e.g., rural versus urban living, road design, vehicle design), and weather factors (e.g., winter driving conditions, particularly in Canada). Loss of the ability to drive affects our ability to participate in and access the community, which can lead to isolation and its inherent consequences.

While much emphasis has been put on whether an older person is fit to drive based on his or her diagnosis or health status, research is necessary to explore what factors affect driving and what can be changed in order to keep this population actively driving in the community. The CIHR Team in Driving in Older Persons (Candrive II) was funded by the Canadian Institutes of Health Research to study older Canadian drivers $(n=928)$ in a multi-centre prospective study across seven sites (Marshall et al., 2013). In addition, the Ozcandrive study, based out of Australia and New Zealand, is following a similar protocol with an additional 250 older drivers. Candrive, which is nearing

* These studies were funded by a Team Grant from the Canadian Institutes of Health Research (CIHR) entitled "The CIHR Team in Driving in Older Persons (Candrive II) Research Program" (grant 90429). Additional support was provided by the Ottawa Hospital Research Institute and the Toronto Rehabilitation Institute, University Health Network. Gary Naglie is supported by the George, Margaret and Gary Hunt Family Chair in Geriatric Medicine, University of Toronto. Michel Bédard was supported by a Canada Research Chair in Aging and Health during the development phase of this study. Ozcandrive was funded by an Australian Research Council Linkage grant (LP 100100078) to Monash University in partnership with La Trobe University, VicRoads, the State of Victoria Department of Justice, the State of Victoria Police, the Transport Accident Commission, Road Safety Trust New Zealand, and Eastern Health.

The Candrive investigators thank Lynn MacLeay, Candrive Program Manager, for her role in managing and operationalizing the study for the Canadian sites. The Candrive investigators also thank the research associates for their dedication and contribution to the success of the study (in order of participant recruitment): Candrive Research Coordinators Jennifer Biggs and Anita Jessup (Ottawa Coordinating Centre); Phyllis McGee (Victoria); Linda Johnson and Joanne Parsons (Winnipeg); Novlette Fraser and Sue Woodard (Toronto); Sheila Garrett (Hamilton); Felice Mendelsohn, Minh-Thy Dinh Truong, Suzie Schwartz, and Rivi Levkovich (Montreal); and Laura Morrison and Hillary Maxwell (Thunder Bay). Many thanks to the additional support staff for their dedication and contribution to the success of the study: Kelly Weegar and Chantal Rockwell (Ottawa).

The Candrive investigators also thank the Candrive cohort study participants for their dedication. Without their commitment, this publication would not have been possible. 
its conclusion, was designed to answer many questions regarding older drivers, providing information on their driving patterns and changes in driving over time, as well as how their health and vehicle choices affect their driving. The articles in this special issue address topics relevant to older Canadian drivers, with the ultimate aim of extending the safe driving period for this population.

\section{Objectives of Special Issue}

The objective of this special issue is to highlight older driver research that is relevant to Canadians. This research reflects the complex elements and factors involved in making decisions about driving fitness, as well as policies and environmental design that can influence older driver safety. The aim is to demonstrate the necessary interplay between driver health and health changes over time, driver attitudes and behaviour, health care interventions, and driving environments including weather and vehicles, as well as the impact of policy concerning driver and public safety.

\section{Organization of Special Issue}

This special issue focuses on older driver research. Seven of the 10 articles are based directly on Candrive/ Ozcandrive (Marshall, 2013) data. The three remaining articles are directly relevant to older drivers and were written by Candrive team members. Gagnon et al. establish the relevance and similarity of the Candrive study cohort to the older Canadian driving population by comparison with the Canadian Community Health Survey - Healthy Aging (CCHS-HA) (Statistics Canada, 2010). This is relevant to the results of the Candrive study since it suggests that the findings may be reflective of the older Canadian driving population. Given the prospective nature of the Candrive study, Jouk et al. were able to explore such topics as the impact of change in attitude and confidence and its effect on driving patterns. From the opposite perspective, Rapoport et al. and Tuokko et al. examine the effects of change in cognition and overall health, respectively, on driving confidence and beliefs among older drivers. In their systematic review, Cameron and Rapoport show that the use of antidepressant medications potentially contributes to a significant increased risk of collision involvement in older drivers.

One of the advantages of the Candrive/Ozcandrive studies is that, not only could subjective information on driver attitudes and behaviours be collected, but also electronic GPS monitoring could formally confirm driving patterns. Smith et al. explore the effects of weather on driving and demonstrate that weather and daylight influence driving patterns but other factors are also likely at play in relation to driving patterns. Similarly, Koppel et al. further use direct driver observation to confirm that driving ability is related to participants' perceived driving abilities and reported frequency of driving in challenging situations. Vrkljan et al. and Kajaks et al. explore the interaction between driver and vehicle. Vrkljan et al. confirm that driver size and gender influence the type and size of vehicle purchased. In a novel simulation, Kajaks et al. are able to show that reduced neck range of motion, often present in older drivers, can influence their ability to check the left blind spot, which has direct implications for vehicle design.

This research reinforces that multiple factors contribute to driving safety. It is this complexity that leads to confusion and uncertainty when drivers, families, and health care practitioners must work together to consider the possibility of driving cessation in relation to health issues. These challenges are nicely summarized by Mazer et al., who examine the ethics of reporting health-related driving risk to licensing authorities.

\section{Concluding Remarks}

Multiple facets of driving, from the road conditions to the vehicles and even the drivers, have continued to change and evolve over the years. While overall road safety continues to improve, ongoing research into older driver issues remains important as this driver population is the fastest growing. Factors such as health and the anticipation of driving cessation are particularly relevant to older drivers and need further study as part of the process of extending the safe driving period for older people.

Shawn C. Marshall

The Ottawa Hospital Research Institute

Guest Editor

\section{References}

Charlton, J., Koppel, S., Odell, M., Devlin, A., Langford, J., O'Hare, M., et al. (2010). Influence of chronic illness on crash involvement of motor vehicle drivers ( 2 nd ed.). Report no. 300. Melbourne, Australia: Monash University Accident Research Centre.

Marshall, S. C. (2008). The role of reduced fitness to drive due to medical impairments in explaining crashes involving older drivers. Traffic Injury Prevention, 9, 291-298.

Marshall, S. C., Man-Son-Hing, M., Bédard, M., Charlton, J., Gagnon, S., Gélinas, I., et al. (2013). Protocol for Candrive II/Ozcandrive, a multicentre prospective older driver cohort study. Accident Analysis \& Prevention (Candrive special issue), 61, 245-252.

Statistics Canada (2010). Canadian Community Health Survey CCHS HA-Healthy Aging (CCHS). User Guide. 
Ottawa, ON: Author. Retrieved from http://www23. statcan.gc.ca/imdb/p2SV.pl?Function=getSurvey\&SD DS $=5146$

Turcotte, M. (2012). Profile of seniors' transportation habits. In Canadian Social Trends (component of Statistics Canada Catalogue no.11-008). Ottawa, ON: Statistics Canada. http: / / www.statcan.gc.ca/pub/11-008-x/2012001/ article/11619-eng.pdf

World Health Organization (2013). How to use the ICF: A practical manual for using the International Classification of Functioning, Disability and Health (ICF). (Exposure draft for comment). Geneva, Switzerland: Author. 